\title{
OS DESAFIOS DA METROLOGIA POR COORDENADAS NOS PROCESSOS DE CONTROLO DAS ESPECIFICAÇÕES DIMENSIONAIS E GEOMÉTRICAS DE COMPONENTES TÉCNICOS, NO NOVO PARADIGMA DA INDÚSTRIA 4.0
}

\author{
F. A. M. Ferreira ${ }^{*}$; H. A. G. Guerra ${ }^{1}$ \\ 1 CATIM - Centro de Apoio Tecnológico à Indústria Metalomecânica, Porto, Portugal \\ *fernando.ferreira@catim.pt
}

Artigo submetido em 15/01/2018 e aceito em 05/03/2018

\begin{abstract}
RESUMO
Produzir componentes técnicos de grande complexidade e com elevado número de especificações, exige que as empresas possuam meios de controlo expeditos que permitam realizar a avaliação da conformidade. Neste contexto, a metrologia revela-se um importante recurso para apoio aos processos de inovação e desenvolvimento e aos processos produtivos, e permite acompanhar o progresso tecnológico de acordo com os principios da indústria 4.0. Nas empresas, a necessidade de garantir a fiabilidade do processo produtivo, garantindo conformidade dimensional e geométrica dos componentes
\end{abstract}

técnicos, exige à metrologia recursos técnicos cada vez mais sofisticados, garantindo um bom desempenho da função metrológica. Perante a necessidade de garantir conformidade das especificações dimensionais e geométricas dos componentes técnicos, estas podem ser controladas com máquinas de medição por coordenadas, tecnologia que promove controlos rápidos e com capacidade para gerar um grande volume de dados, facilitando a interacção com o processo produtivo, procurando garantir a sua conformidade.

PALAVRAS-CHAVE: fábricas inteligentes, inovação, desenvolvimento tecnológico, metrologia, especificações dimensionais e geométricas, máquinas de medição por coordenadas, indústria 4.0.

\section{THE CHALLENGES OF COORDINATE METROLOGY IN THE CONTROL OF DIMENSIONAL AND GEOMETRICAL SPECIFICATIONS OF TECHNICAL COMPONENTS IN THE NEW PARADIGM OF THE INDUSTRY 4.0}

\begin{abstract}
The production of highly complex technical components with a large number of specifications requires companies to have expeditious means of control to carry out the conformity assessment. In this context, the metrology is an important resource in support of innovation and development processes and production processes, and allows monitoring of technological progress according to the principles of industry 4.0. In companies, the need to guarantee the reliability of the production process, ensuring dimensional and geometrical compliance of the
\end{abstract}

technical components, requires to metrology technical resources increasingly sophisticated, able to ensuring a good performance of the metrological function. Given the need to ensure compliance of the dimensional and geometrical specifications of the technical components, these can be controlled with coordinate measuring machines, a technology that promotes rapid controls and with the capacity to generate a large volume of data, facilitating interaction with the production process, seeking to guarantee their conformity.

KEYWORDS: smart factories, innovation, technological development, metrology, dimensional and geometrical specifications, coordinate measuring machines, industry 4.0 . 


\section{INTRODUÇÃO}

A inovação e o desenvolvimento científico e tecnológico têm sido, ao longo do tempo, importantes mecanismos para a optimização de processos, melhoria da eficiência e aumento da competitividade e sustentabilidade das organizações.

“A Indústria 4.0 não é uma corrente ou uma moda, mas sim uma evolução dos sistemas produtivos industriais que garante benefícios como a redução de custos, de energia, o aumento da segurança e da qualidade, e a melhoria da eficiência dos processos" (Ribas, 2017).

No caso particular da indústria, a competitividade natural entre empresas tem acelerado os processos de inovação e desenvolvimento e exigido simultaneamente que os sistemas de produção e de controlo da qualidade apresentem rapidez, flexibilidade e fiabilidade. Como tal, a introdução das tecnologias digitais e a intensificação dos processos de inovação tem proporcionado uma redução dos tempos de desenvolvimento, dos tempos de produção e dos prazos de introdução de novos produtos no mercado.

\section{a) As "Fábricas Inteligentes"}

No caso das denominadas "Fábricas Inteligentes", fábricas onde todos os intervenientes do processo produtivo de um componente técnico estão ligados a uma base de dados "Big Data" onde se pode consultar e analisar todos os dados referentes à produção de um componente técnico, e intervir se necessário nas máquinas de produção sem intervenção humana, estas tem adoptado estratégias de introdução das mais recentes tecnologias digitais e intensificado os processos de inovação, design e desenvolvimento, estimulando dessa forma uma crescente produção de produtos e componentes técnicos com geometrias cada vez mais complexas e sofisticadas, que se evidenciam nos diversos mercados e contribuem para manter a liderança em muitas áreas da indústria mundial.

b) A produção de peças e componentes técnicos de grande complexidade

No entanto, produzir peças e componentes técnicos de grande complexidade e com elevado número de especificações, em muitos casos em processos produtivos que estão sujeitos a uma variedade de factores que de forma mais ou menos significativa afectam as especificações estabelecidas colocando em causa a qualidade do produto final, exige que as empresas tenham uma clara implementação da função metrológica e meios de controlo expeditos que lhes permitam realizar com segurança e fiabilidade a avaliação da sua conformidade metrológica, garantindo assim a funcionalidade, a segurança e a estética.

c) A metrologia, ciência da medição

Ao longo do tempo a metrologia, enquanto ciência da medição apresentou-se sempre como uma das áreas do conhecimento científico que maior aplicabilidade tem nos meios industriais, revelando-se essencial para a construção de conhecimento em diversas áreas, contribuindo para a formação e desenvolvimento intelectual, estruturação do pensamento e raciocínio dedutivo de forma a intervir nos processos procurando minimizar todos os aspectos negativos associados aos processos produtivos, passando assim a fornecer suporte essencial para avaliação da conformidade de processos e produtos, sendo por isso um importante pilar da qualidade.

Ora, se a metrologia tem, por um lado e em termos mais gerais a função de garantir relações comerciais justas, promover a cidadania e assegurar o reconhecimento nacional e internacional, tem por outro lado a necessidade de fornecer cada vez maior e melhor suporte ao controlo das caracteristicas dos produtos, reforçando assim a importância que representa para os sistemas da qualidade das organizações. 


\section{d) Metrologia 4.0 para o novo paradigma industrial}

Considerando que por enquanto é apenas possivel colocar este trabalho num plano conceptual, a questão que se coloca na actualidade é quais os meios técnicos que permitem à metrologia, no caso particular da metrologia dimensional e geométrica, acompanhar e dar suporte ao processo tecnológico de acordo com os princípios da indústria 4.0?

\section{A METROLOGIA POR COORDENADAS E OS DESAFIOS DA INDÚSTRIA 4.0}

A indústria 4.0, para além de um termo que integra várias tecnologias e concepções de organização em cadeia com base nos conceitos tecnológicos de sistemas "Cyber-physical", "Internet of Things", "Big Data", "Smart Factory", etc., é um projecto de alta tecnologia com origem na Alemanha, que tem como principal objectivo transformar os processos produtivos tradicionais automatizando-os.

As tecnologias associadas à indústria 4.0 proporcionam $\mathrm{o}$ fabrico de produtos $\mathrm{e}$ fornecimento de serviços cada vez mais inteligentes, a promoção de uma relação eficaz entre as partes interessadas, a intensificação dos processos de inovação e desenvolvimento, o aumento da produtividade, a melhoria do planeamento, a acção preventiva em tempo real através de plataformas digitais que conectam tecnologias de recolha de dados e informação com tecnologias operativas de forma a optimizar processos.

Esta nova realidade industrial procura, através da integração de novos recursos tecnológicos de interacção entre máquina-máquina e máquina-operador, optimizar os processos produtivos tornando-os eficientes, contribuindo assim para uma melhor gestão dos recursos nas organizações e consequentemente para um crescimento da própria economia.

A alavancar esta nova realidade industrial está a conectividade digital que é possivel estabelecer entre as diferentes máquinas de produção e de controlo existentes nas fábricas, que uma vez ligadas em rede através de plataformas digitais geram e partilham grande volume de dados de informação em tempo real, passando a informação em formato convencional (papel) a assumir uma função de menor relevo nas organizações.

\section{1) Integração de processos na indústria 4.0}

No contexto da realidade da indústria 4.0, e considerando como fundamental que todos os intervenientes do processo produtivo e do processo de controlo de qualidade de um componente técnico estão ligados a uma base de dados onde podem consultar e analisar os dados referentes à produção desse mesmo componente, é expectável que os dados gerados e a informação obtida permitam eventuais intervenções nas máquinas de produção, alterando parâmetros que possibilitem a produção de componentes técnicos que cumpram com as especificações estabelecidas, sem ser necessário recorrer a intervenção humana. 


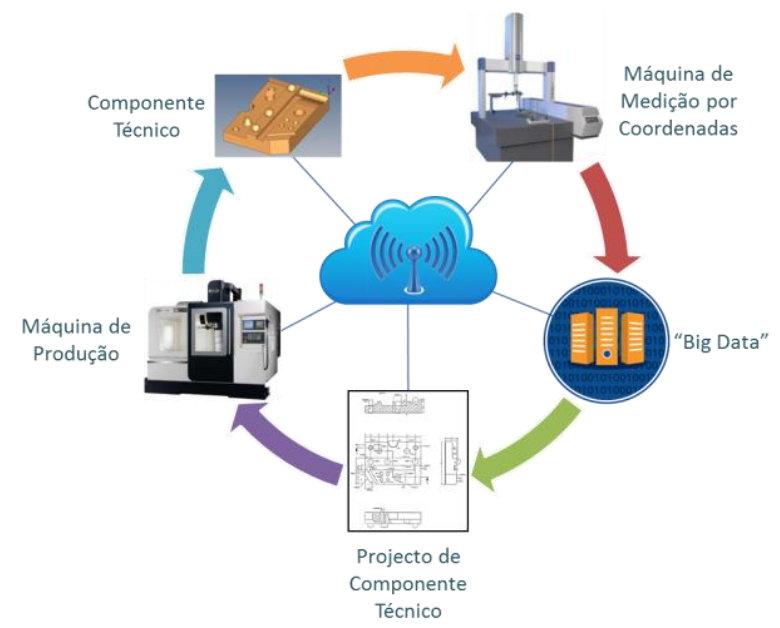

Figura 1 - Processo de integração (processo produtivo e controlo da qualidade) na indústria 4.0 Fonte: Elaborado pelos autores (2017).

2.2) A metrologia e a função metrológica na indústria

A necessidade de garantir a fiabilidade do processo produtivo, garantindo a conformidade dimensional e geométrica dos componentes técnicos produzidos nos exigentes sectores da indústria automóvel, aeronáutica, naval e eólica, exige da metrologia recursos cada vez mais sofisticados, capazes de garantir um bom desempenho da função metrológica e simultaneamente uma boa estabilidade metrológica dos componentes técnicos produzidos.

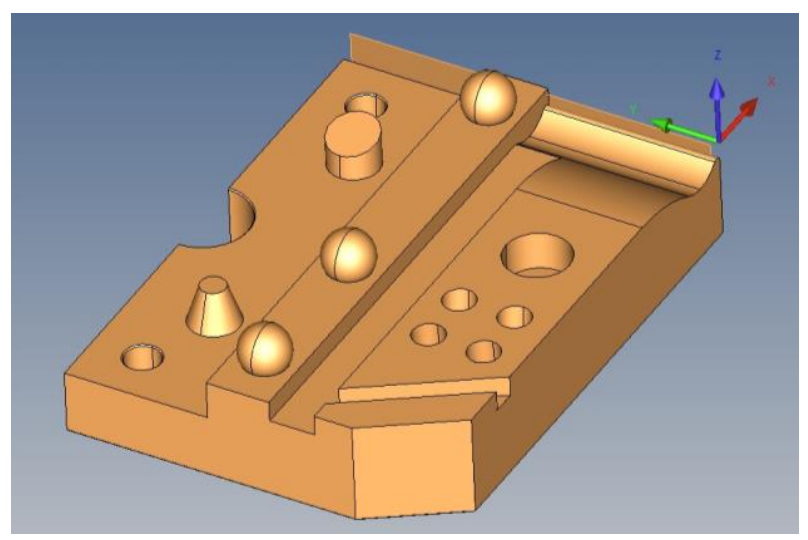

Figura 2 - Exemplo de componente técnico.

Fonte: Ferreira et al.(2013)

Tal como os processos produtivos, os processos de medição em ambientes industriais, apresentam susceptibilidade a factores como condições ambientais, exactidão dos instrumentos de medição, etc. que influenciam também o cumprimento das especificações técnicas dos produtos finais. No caso da metrologia dimensional e geométrica, o suporte que esta fornece ao processo produtivo no controlo das especificações técnicas dos produtos, a maior parte dos equipamentos convencionais de medição (paquímetros, micrómetros, comparadores, sutas, etc.) revelam-se insuficientes, pouco versáteis e não permitem a realização de ensaios complexos e eventual recolha de grande volume de dados que caracterizem de forma exacta cada caracteristica a avaliar.

Para analisar de forma detalhada a complexidade da geometria dos componentes técnicos produzidos, o que implica a análise de grande quantidade de dados que caracterizam cada caracteristica dimensional ou geométrica desse mesmo componente, é frequente a utilização de máquinas de medição por coordenadas. As máquinas de medição por coordenadas, enquanto 
sofisticados sistemas de medição, permitem obter coordenadas de diversos pontos de um componente técnico para posterior "construção" dos elementos geométricos que o constituem e respectiva avaliação dimensional e geométrica.

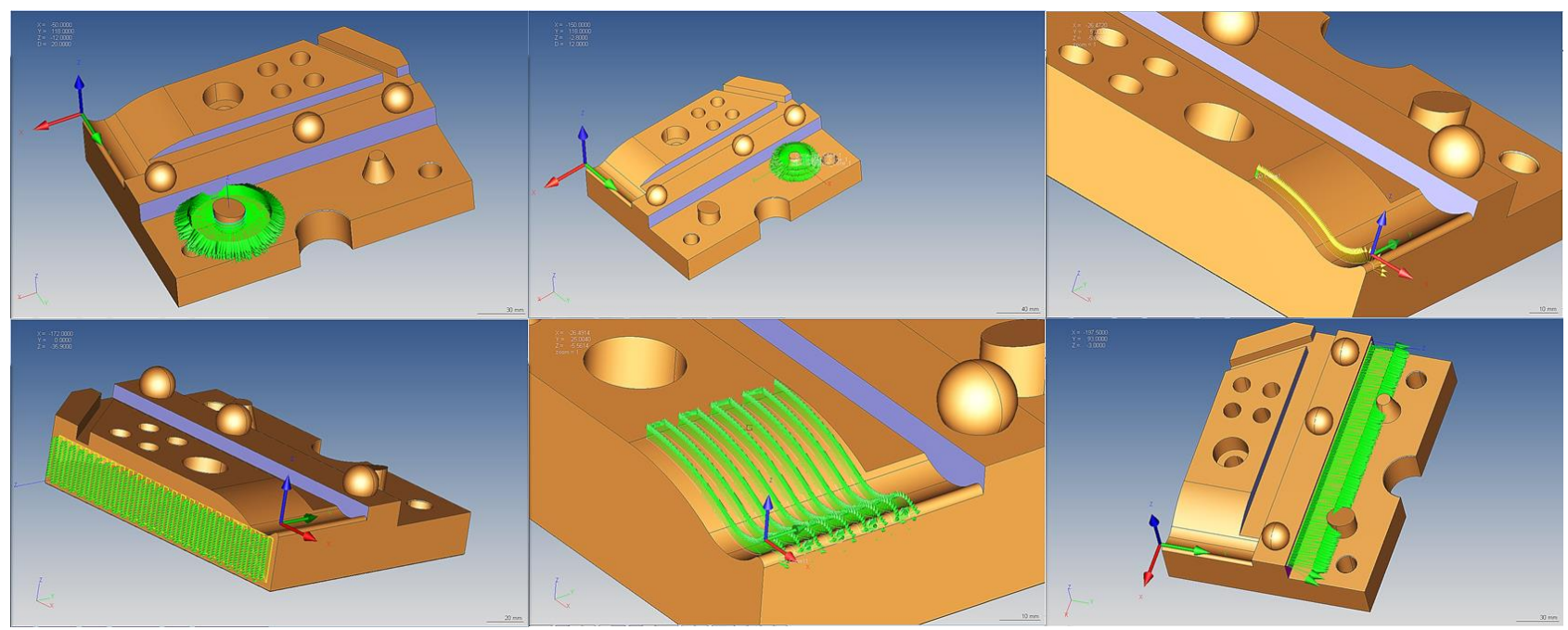

Figura 3 - Exemplos de diferentes elementos geométricos a controlar em componentes técnicos Fonte: Elaborado pelos autores (2017).

De acordo com as exigencias definidas no projecto de cada componente técnico, elementos geométricos como a linha, o plano, o cilindro, o cone, a esfera, são construidos para análise das zonas identificadas nos desenhos técnicos e avaliação da conformidade.

\section{3) Dimensões e geometrias em elementos geométricos de componentes técnicos}

Em elementos geométricos, como a linha, o plano, o cilindro, o cone e a esfera, é possivel realizar diferentes tipos de avaliação. Por um lado é possivel quantificar a dimensão de cada um dos elementos geométricos, por outro lado é também possivel avaliar a geometria e os diferentes erros de forma (rectitude, planeza, circularidade, cilindricidade, perfil de linha e perfil de superfície), de orientação (paralelismo, perpendicularidade e angularidade), de posição (localização, concentricidade, coaxialidade e simetria) e de batimento (batimento axial, batimento axial total, batimento radial e batimento radial total) que apresentam.

No caso particular da avaliação de um circulo, considerando a vasta informação que é possivel recolher (grande número de pontos) é possivel avaliar o diâmetro e até o posicionamento do seu centro em relação ao referencial, mas também é possivel avaliar as caracteristicas geométricas como, por exemplo, a circularidade. Ou seja, o quanto se aproxima ou afasta de um circulo perfeito. 


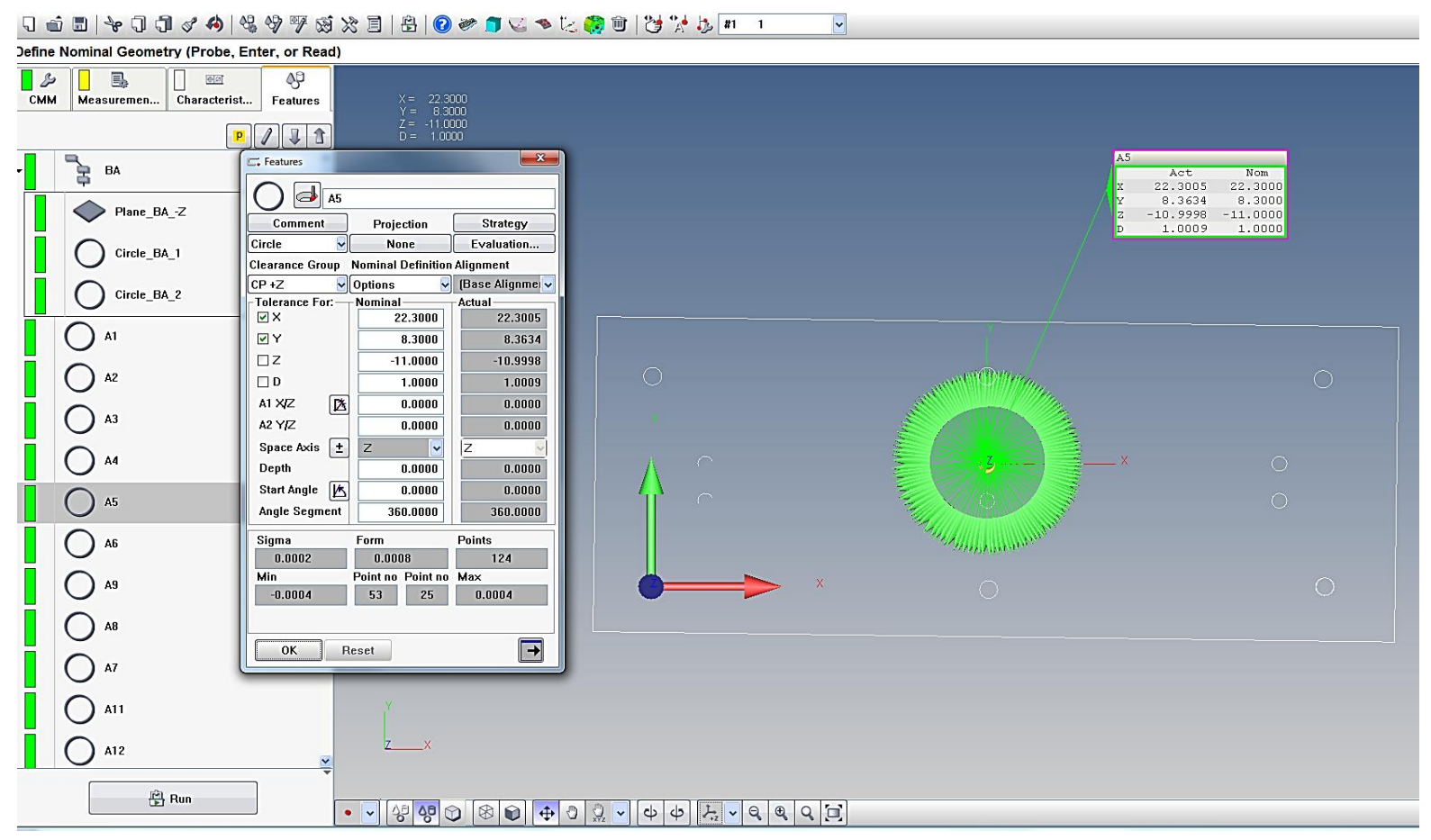

Figura 4 - Elemento geométrico (circulo) e possiveis caracteristicas a avaliar em máquina de medição por coordenadas de marca ZEISS e software Calypso (diâmetro, posicionamento e circularidade)

Fonte: Elaborado pelos autores (2017).

2.4) As máquinas de medição por coordenadas

Perante a necessidade de garantir conformidade de diferentes especificações dimensionais e geométricas dos componentes técnicos produzidos, estes podem ser medidos com o mais versátil e importante recurso - as máquinas de medição por coordenadas 3D.

As máquinas de medição por coordenadas, durante um processo de ensaio dimensional e geométrico, são capazes de gerar grande volume de dados com informação referente ao estado de cada caracteristica controlada, de enviar esses dados para servidores onde serão processados e analisados para eventual interacção com o processo produtivo e reprogramação de parâmetros de máquinas de produção quando necessário, ou seja, cumprindo com as exigências, conceitos e objectivos da indústria 4.0 . 


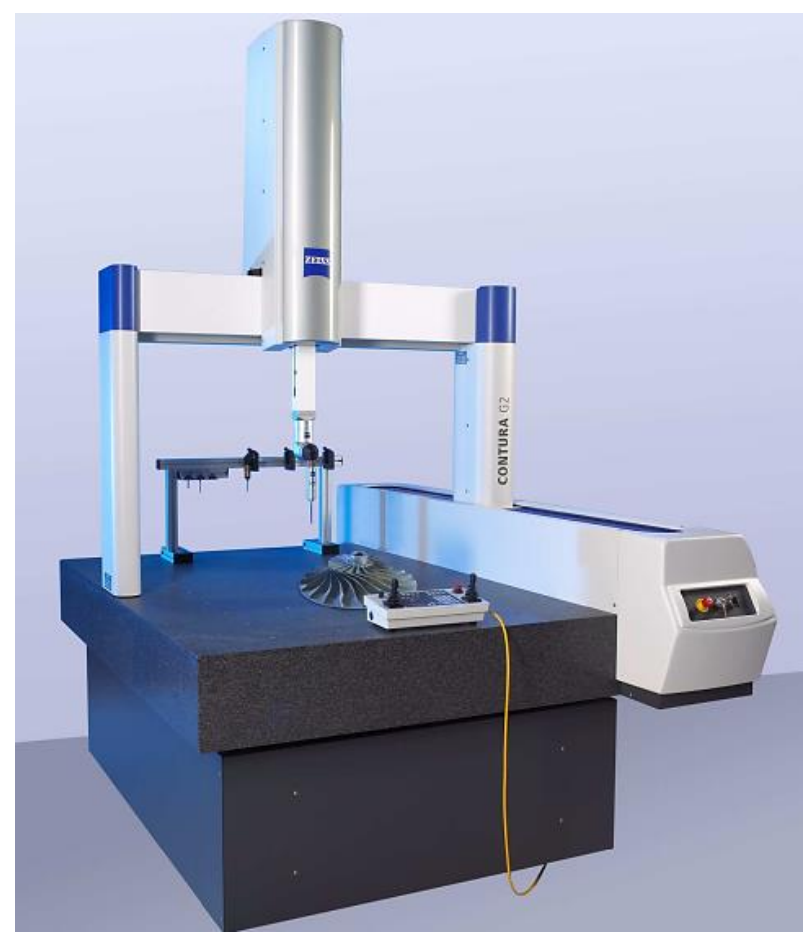

Figura 5 - Máquina de medição por coordenadas de marca ZEISS e modelo Contura G2 (site 1, acedido em 8 de dezembro de 2017)

"As máquinas de medição por coordenadas vieram dar um novo impulso no campo da metrologia geométrica e dimensional. As máquinas de medição por coordenadas em ambientes industriais tornou-se um importante recurso para os sistemas de qualidade, para a monitorização de processos de fabricação, na redução de erros durante o processo de fabrico, inspecção de especificações de produtos e melhoria contínua da qualidade" (Ferreira et al., 2013)

As máquinas de medição por coordenadas 3D apresentam-se pois como sistemas de medição sofisticados e flexiveis, com boa repetibilidade e reprodutibilidade nas medições, que permitem efectuar medições recorrendo a diferentes tipos de sensores de contacto ou sistemas ópticos, e acompanham a efectividade dos mais recentes sistemas de produção de acordo com as exigências da indústria 4.0, o novo paradigma das empresas.

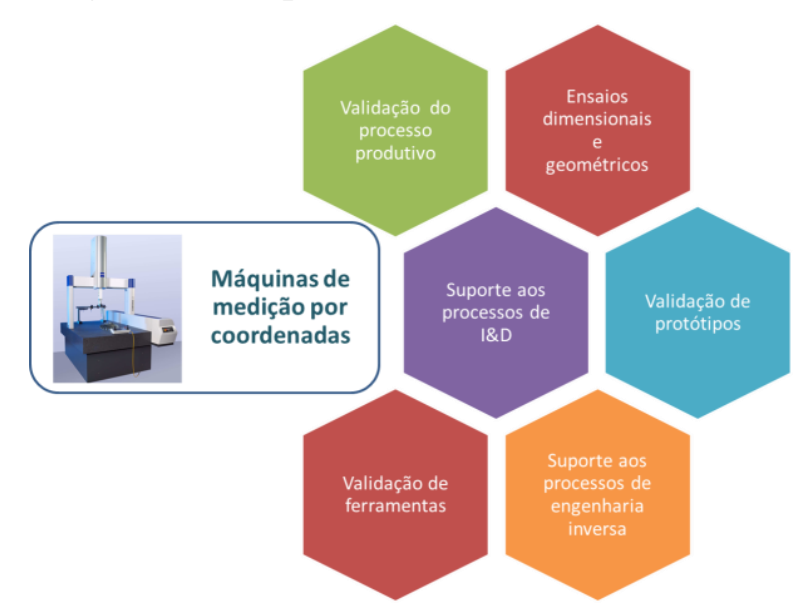

Figura 6 - Suporte das máquinas de medição por coordenadas a diferentes tipos de processos

Fonte: Elaborado pelos autores (2014).

Estes sistemas de medição, importantes no apoio aos processos de tomada de decisão, apresentam incertezas de medição relativamente baixas e são sujeitos a processos de calibração 
acreditada de acordo com os requisitos do referencial normativo ISO 10360 (2009), assegurando desta forma a rastreabilidade metrológica.

\section{CONCLUSÃO}

A capacidade de inovação, ou a capacidade de disponibilizar recursos para implementar inovações em processos, tecnologias ou outros, rapidamente e com eficácia e eficiência, mas sem criar custos acrescidos, depende muito do conhecimento obtido na avaliação de recursos existentes nas organizações.

No caso particular da metrologia e no papel que esta desempenha nas empresas, ou seja, os desafios da metrologia por coordenadas nos processos de controlo das especificações dimensionais e geométricas de componentes técnicos, no novo paradigma da indústria 4.0, é absolutamente imprescindível que exista uma integração das máquinas de medição por coordenadas no suporte aos processos produtivo.

Dos diferentes sistemas de medição existentes, aqueles que pelas suas caracteristicas e capacidades mais alinhados estão com os conceitos da indústria 4.0, são as máquinas de medição por coordenadas. Estas utilizam tecnologia que promove a realização de ensaios dimensionais e geométricos relativamente rápidos, capazes de adquirir um grande volume de dados capazes de caracterizar correctamente cada elemento geométrico em avaliação, o que potencia a integração destes sistemas de medição nas linhas de produção onde equipamentos robotizados podem alimentar as máquinas de medição por coordenadas de forma contínua (sem necessária intervenção humana permanente), potenciando assim a interacção eficiente entre o processo produtivo e o processo de controlo da qualidade, procurando garantir a conformidade dos componentes técnicos produzidos.

\section{REFERENCIAS}

FERREIRA, F., VICENTE, J., PEREZ, S. (2013) Evaluation of coordinate measuring machines in the industry, using calibrated artefacts. The Manufacturing Engineering Society International Conference, MESIC 2013, Procedia Engineering v.63, p.659-668, 2013.

ISO 10360-2. Geometrical product specifications (GPS) - Acceptance and reverification tests for coordinate measuring machines (CMM) - Part 2, 2009.

RIBAS, C. Indústria 4.0, a quarta revolução industrial. O Jornal Económico, Julho, Portugal, 2017. 